\title{
Extinction and polarization of radiation by absorbing spheroids: shape/size effects and benchmark results ${ }^{1}$
}

\author{
N.V. Voshchinnikov ${ }^{\mathrm{a}, 2}$ V.B. Il'in ${ }^{\mathrm{a}}$ Th. Henning ${ }^{\mathrm{b}}$ B. Michel ${ }^{\mathrm{b}}$ \\ V.G. Farafonov ${ }^{\mathrm{c}}$ \\ a Sobolev Astronomical Institute, St. Petersburg University, \\ St. Petersburg-Peterhof, 198904 Russia \\ ${ }^{\mathrm{b}}$ Astrophysical Institute and University Observatory, Fr. Schiller University, \\ Schillergäßchen 2-3, D-07745 Jena, Germany \\ ${ }^{c}$ St. Petersburg University of Aerocosmic Instrumentation, St. Petersburg, 190000 \\ Russia \\ (Received 10 September 1998)
}

\begin{abstract}
We use the separation of variables and T-matrix methods to calculate the optical properties of homogeneous spheroids with refractive indices from $m=1.3+0.0 i$ up to $3+4 i$.

It is found that the extinction cross-sections for highly absorbing spheroids are normally $1.5-2$ times larger than those for spheres of the same volume. The albedo of the non-spherical particles rather slightly depends on the particle shape and is mainly determined by the imaginary part of the refractive index. Beginning at some size, the spheroidal particles do not polarize the transmitted radiation independent of their shape.

We also suggest a new approach for axisymmetric particles which would combine the strong aspects of both methods mentioned above and give several values of the cross-sections as benchmarks in tabular form.
\end{abstract}

Key words: Light scattering; Non-spherical particles; Polarization; Benchmarks

$\overline{1}$ Accepted for publication in Journal of Quantative Spectroscopy \& Radiat. Transfer

2 To whom all correspondence should be addressed. Phone: $(+7)$ 812/428 42 63; Fax: (+7) 812/428 71 29; e-mail:nvv@aisbpu.spb.su 


\section{Introduction}

In many scientific and engineering applications prolate and oblate spheroids are appropriate models for real particles. The scattering of electromagnetic radiation by spheroids is usually calculated by the separation of variables method (11; 2) (SVM) and the T-matrix method (3) (TMM). Although both approaches are well-suited for numerical computations, it is often difficult to give reliable error estimates. For non-absorbing particles, there always exists an internal check as the absorption cross-sections (and efficiency factors) must be equal to zero. Obviously, for particles consisting of absorbing materials like silicates, graphite or metals, this test does not work. Despite the fact that rather elaborated methods for the calculation of the optical properties of highly absorbing non-spherical particles can be found in literature (see, for example, Michel et al. (4)), these methods have never been used for detailed numerical studies.

In this paper, we consider the light scattering by homogeneous spheroids using the most popular approaches - SVM and TMM. The extinction and polarization cross-sections for prolate and oblate spheroids of various aspect ratios $a / b$ are calculated for the refractive indices $m$ typical of soot, graphite, iron, silicate, and water ice and compared with the results for the particles of the same volume. A part of the results is presented in tabular form serving as benchmarks for forthcoming calculations. We also describe a combined approach to calculate the light scattering by axisymmetric particles. The method should allow to treat easily (very) elongated and flattened absorbing particles.

\section{Description of methods}

\subsection{General definitions}

A spheroid (ellipsoid of revolution) is obtained by the rotation of an ellipse around its major axis (prolate spheroid) or its minor axis (oblate spheroid). The ratio of the major semiaxis $a$ to the minor semiaxis $b$ (i.e. the aspect ratio

$a / b)$ characterizes the particle shape which may vary from a nearly spherical one $(a / b \approx 1)$ to a needle or a disk $(a / b \gg 1)$.

We assume an incident plane wave having the wavelength $\lambda$. Let $\alpha$ denote the angle between the propagation direction and the rotation axis of the spheroid $\left(0^{\circ} \leq \alpha \leq 90^{\circ}\right)$

For axial propagation $\left(\alpha=0^{\circ}\right)$, there is no polarization of transmitted radi- 
ation due to symmetry. If $\alpha \neq 0^{\circ}$, two cases of polarization of the incident radiation have to be considered: the electric vector $\vec{E}$ is parallel (TM mode) or perpendicular (TE mode) to the plane defined by the spheroid's rotation axis and the wave propagation vector.

The size parameter is given by

$$
x_{\mathrm{V}}=\frac{2 \pi r_{\mathrm{V}}}{\lambda}
$$

where $r_{\mathrm{V}}$ is the radius of the sphere whose volume is equal to that of the spheroid. The radius $r_{\mathrm{V}}$ is defined as

$$
r_{\mathrm{V}}^{3}=a b^{2} \quad \text { for prolate spheroids }
$$

and

$$
r_{\mathrm{V}}^{3}=a^{2} b \quad \text { for oblate spheroids. }
$$

One usually calculates the efficiency factors $Q=C / G$ which are the ratio of the corresponding cross-sections $C$ to the geometrical cross-section $G$ of the spheroid (the area of the particle's shadow)

$$
G(\alpha)=\pi b\left(a^{2} \sin ^{2} \alpha+b^{2} \cos ^{2} \alpha\right)^{1 / 2} \quad \text { for prolate spheroids }
$$

and

$$
G(\alpha)=\pi a\left(a^{2} \cos ^{2} \alpha+b^{2} \sin ^{2} \alpha\right)^{1 / 2} \quad \text { for oblate spheroids. }
$$

In order to compare the optical properties of the particles of different shapes it is convenient to consider the ratios of the cross-sections for spheroids to the geometrical cross-sections of the equal volume spheres, $C / \pi r_{\mathrm{V}}^{2}$. They can be found as

$$
\frac{C}{\pi r_{\mathrm{V}}^{2}}=\frac{\left[(a / b)^{2} \sin ^{2} \alpha+\cos ^{2} \alpha\right]^{1 / 2}}{(a / b)^{2 / 3}} Q
$$

for a prolate spheroid and

$$
\frac{C}{\pi r_{\mathrm{V}}^{2}}=\frac{\left[(a / b)^{2} \cos ^{2} \alpha+\sin ^{2} \alpha\right]^{1 / 2}}{(a / b)^{1 / 3}} Q
$$

for an oblate spheroid. 
The albedo of a particle can be calculated from the extinction and scattering cross-sections

$$
\Lambda=\frac{C_{s c a}}{C_{e x t}} .
$$

The dichroic polarization efficiency is defined by the extinction cross-sections for TM and TE modes

$$
\frac{P}{\tau}= \pm \frac{C_{e x t}^{\mathrm{TM}}-C_{e x t}^{\mathrm{TE}}}{C_{e x t}^{\mathrm{TM}}+C_{e x t}^{\mathrm{TE}}} \cdot 100 \%,
$$

where the upper (lower) sign is related to prolate (oblate) spheroids. This ratio describes the efficiency of the polarization of light transmitted through the uniform slab consisting of non-rotating particles of the same orientation.

The optical properties of spheroidal particles can be determined by various methods of light scattering theory. Most frequently, the separation of variables method obtained by Asano \& Yamamoto (11) and Farafonov (2; 河) and the Tmatrix method (3; 6) are used. The calculations can be also performed with the discrete dipole approximation (DDA) which can be applied to arbitrarily inhomogeneous and irregular particles, but requires large computation time and is less accurate than the other methods (see discussion in the papers $(7 ;$; $)$ ).

\subsection{Asano 83 Yamamoto's solution for spheroids (SVM1)}

Historically it was the first approach to the light scattering problem for spheroids with a complex refractive index. The method is based on the solution to the Helmholtz equation in the spheroidal coordinate system.

In their pioneering paper, Asano \& Yamamoto (1) used the Debye potentials to describe the electromagnetic fields, which is similar to the Mie solution for spheres. The scattering coefficients then are bound in the infinite systems of the linear algebraic equations and can be found by solving the truncated systems.

In our comparison, we made the calculations with the numerical code of Peter Martin (see (9; 10)). 


\subsection{Farafonov's solution for spheroids (SVM2)}

The principal distinction of this solution (see (2); 11)) from the previous one is the special basis for the representation of the electromagnetic fields - a combination of the Debye and Hertz potentials (i.e. the potentials introduced to solve the light scattering problem for spheres and infinitely long cylinders, respectively).

The approach has an incontestable advantage for strongly elongated or flattened particles. In this paper, the most recent version of our numerical code has been used. It involves different methods to calculate the spheroidal functions with the automatic choice of the most appropriate method among them.

\subsection{Solution for spheroids by the T-matrix method (TMM1 and TMM2)}

Besides SVM, another very popular approach to solve the light scattering problem for spheroids is the T-matrix method. It is mainly applied to the axisymmetric particles: finite cylinders, spheroids, Chebyshev particles.

The main idea of the method is the expansion of the incident, internal and scattered radiation fields in terms of the vector spherical harmonic functions. Because of the linearity of the Maxwell equations, the relations between the expansion coefficients of the fields are linear and given by two matrices. Thus, solution consists of calculations of the matrix elements being the surface integrals and inversion of a matrix. The advantages of the approach are simple compact codes and their high speed. Another advantage is also the ability to treat more the complex case of elastic waves, the potential applicability to particles of complex shape, and the possible analytical averaging over the particle orientations within the expansion coefficients which can greatly enlarge the field of the TMM applications.

We used the T-matrix codes of Barber and Hill (3) (TMM1) and Mishchenko (TMM2; see (6) and references therein).

\subsection{A comparison of methods}

We do not intend here to compare in detail the computational efficiency of different methods. The goal of this subsection is only to illustrate it on one example. A crude impression of the range of applicability of the methods may be obtained from Fig. 1 where the normalized cross-sections are plotted for oblate spheroids with $m=1.7+0.7 i$ and $a / b=4$. In all four cases double 


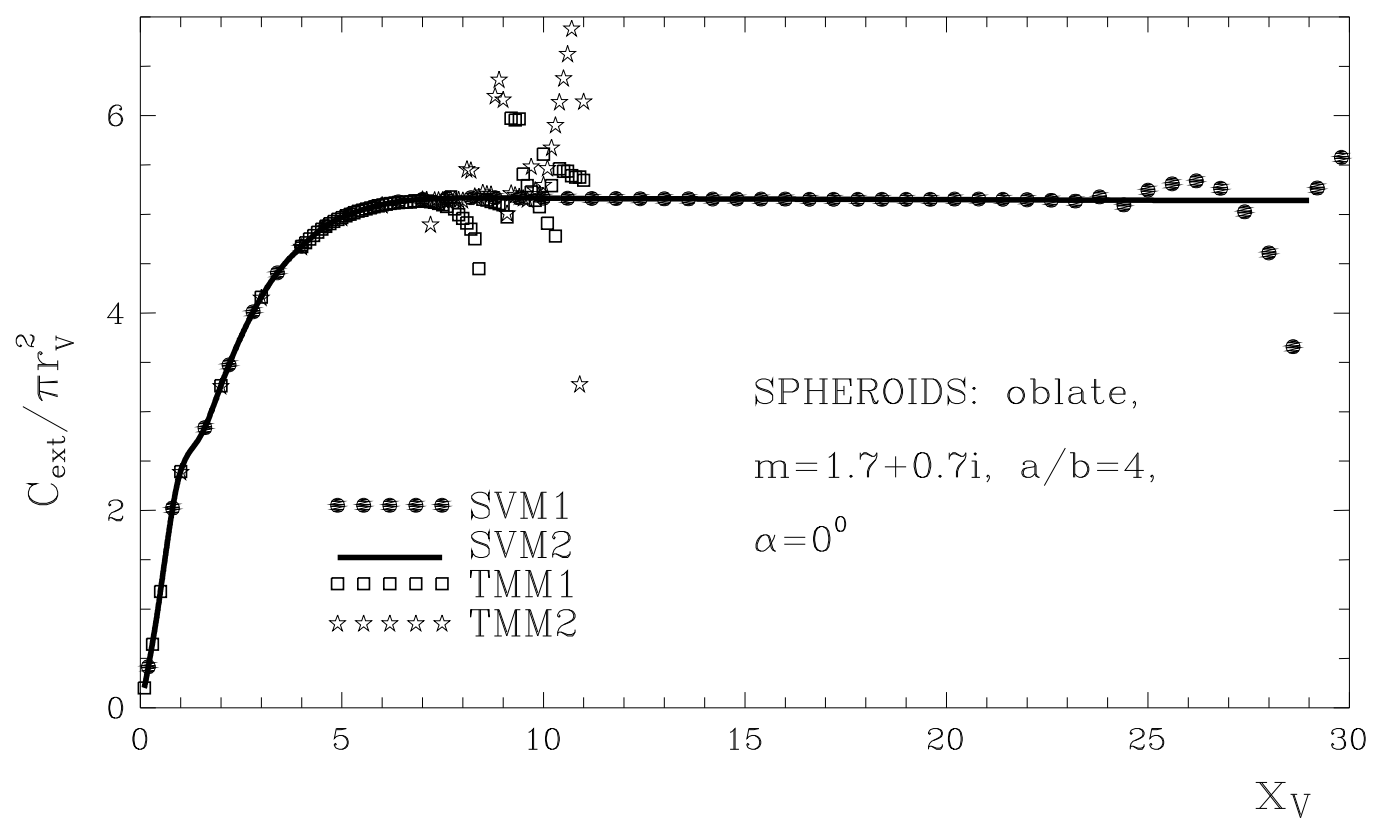

Fig. 1. Normalized extinction cross-sections vs $x_{V}$ for oblate spheroids with the refractive index $m=1.7+0.7 i, a / b=4$ and $\alpha=0^{\circ}$. The solutions of Asano \& Yamamoto (SVM1) and Farafonov (SVM2) and the T-matrix codes of Barber \& Hill (TMM1) and Mishchenko (TMM2) were used in the calculations. Note that because of the special normalization (see Eq. (7)) the cross-sections have the limit $2(a / b)^{2 / 3} \approx 5.040$ if $x_{V} \rightarrow \infty$.

precision was used.

It should be noted that the advantage of the T-matrix codes appears mainly for particles more spherical than presented in the Fig. 1. On the other hand, for larger aspect ratios, the SVM2 code becomes unrivalled.

\subsection{A new method for axisymmetric particles}

Each of the methods (SVM and TMM) has its own problems. We suggest the following approach that could solve some of them.

The incident, scattered and internal fields are divided in two parts: axisymmetric and non-axisymmetric ones

$$
\vec{E}=\vec{E}_{1}+\vec{E}_{2}, \quad \vec{H}=\vec{H}_{1}+\vec{H}_{2}
$$

It is possible to do so that the axisymmetric part does not depend on the azimuthal angle $(\varphi)$ and the averaging of the non-axisymmetric part over all the azimuthal angle values gives zero (2). Then the scattering problem can be 
solved for each of the parts separately.

The scattering problem is formulated in the integral form like in the TMM (e.g., (3)). The Abraham's potentials

$$
p=E_{1 \varphi} \cos \varphi, \quad q=H_{1 \varphi} \cos \varphi
$$

are introduced for the axisymmetric part. A superposition of the Debye and Hertz potentials is used for the non-axisymmetric part:

$$
\vec{E}_{2}=\vec{\nabla} \times\left(U \vec{e}_{z}+V \vec{r}\right), \quad \vec{H}_{2}=\frac{1}{i \mu k_{0}} \vec{\nabla} \times \vec{\nabla} \times\left(U \vec{e}_{z}+V \vec{r}\right)
$$

for TE mode and

$$
\vec{E}_{2}=-\frac{1}{i \varepsilon k_{0}} \vec{\nabla} \times \vec{\nabla} \times\left(U \vec{e}_{z}+V \vec{r}\right), \quad \vec{H}_{2}=\vec{\nabla} \times\left(U \vec{e}_{z}+V \vec{r}\right)
$$

for TM mode. Here $\varepsilon$ is the complex permittivity, $\mu$ the magnetic permeability,

$k_{0}$ the wavevector in vacuum, $\vec{r}$ the radius-vector, $\vec{e}_{z}$ the unit vector, and $i$ the complex unity.

The scalar potentials are expanded in terms of the spherical wave functions. The coefficients of the expansions are determined from solution to the algebraic systems similar to those obtained in the TMM (e.g., (3)).

Thus, the new approach should combine the strong aspects of the basic methods: the simple solution scheme typical of the TMM and the ability to treat particles whose shape can be very elongated or flattened as in the SVM2. The theoretical description of this approach is given in (12) the development of a numerical code realizing it is in progress (13).

\section{Numerical results}

Here we present some results illustrating the behaviour of the optical properties of spheroidal particles for the case of transmitted radiation. Because this is the first consideration of optical properties of highly absorbing non-spherical particles beyond the Rayleigh limit, we try to demonstrate the features of such particles in extinction, scattering and polarization.

Note that in the standard case of light scattering by spheres (Mie theory) only the refractive index and the size parameter of particles may be varied. For the homogeneous spheroids, one can also study the effects of the particle 
shape (the aspect ratio $a / b$ ) and orientation (the angle $\alpha$ ) as well as that of the polarization state of the incident radiation (TM and TE modes) for both elongated (prolate spheroids) and flattened (oblate spheroids) particles.

We consider spheroids with aspect ratios $a / b=1.1,2,4$, and 10 in a fixed orientation. The first $a / b$ value illustrates the influence of small deviations from the spherical shape. All the calculations presented in this Section were performed using our new SVM2 code.

\subsection{Extinction}

Figures 2 and 3 show the normalized extinction cross-sections for non-absorbing and absorbing spheroids with different aspect ratios. The similar scale of the $y$-axes allows to see clearly the shape effects for the particles of equal volume.

Note that large oscillations and the ripple-like structure disappear for absorbing spheroids - the curves become very smooth. These features depend mainly on the value of the imaginary part of the refractive index. The curves $C_{\text {ext }} / \pi r_{V}^{2}(x)$ for $\alpha=0^{\circ}$ and $\alpha=90^{\circ}$ differ more and more when the ratio $a / b$ grows (Fig. 3). For absorbing spheroids, the position of the first (main) maximum slightly shifts to larger values of $x_{V}$ with increasing $a / b$, while for non-absorbing particles, we have the opposite case.

Now let us consider the particle of a fixed volume and begin to change its shape from a sphere to a needle or disk. The extinction by the particle is shown in Fig. 4 for three values of the refractive index. As follows from this Figure, in almost all cases the extinction cross-sections seem to reach their asymptotic values already at $a / b \sim 10$. It is interesting that the extinction by non-spherical transparent particles is usually smaller than that by spheres. The absorbing spheroids normally remove from the incident beam $1.5-2$ times more energy than spheres of the same volume. Though the behaviour of $C_{\text {ext }}$ is refractive index/size dependent, the conclusions we made are rather general for absorbing spheroids. From Figs. 3, 4 one can also see that even after averaging over particle's orientations we should get a stronger extinction for particles with larger aspect ratios $a / b$.

\subsection{Scattering and albedo}

If the imaginary part of the refractive index is not zero, we can study independently the scattering and absorbing properties of particles. In the case 

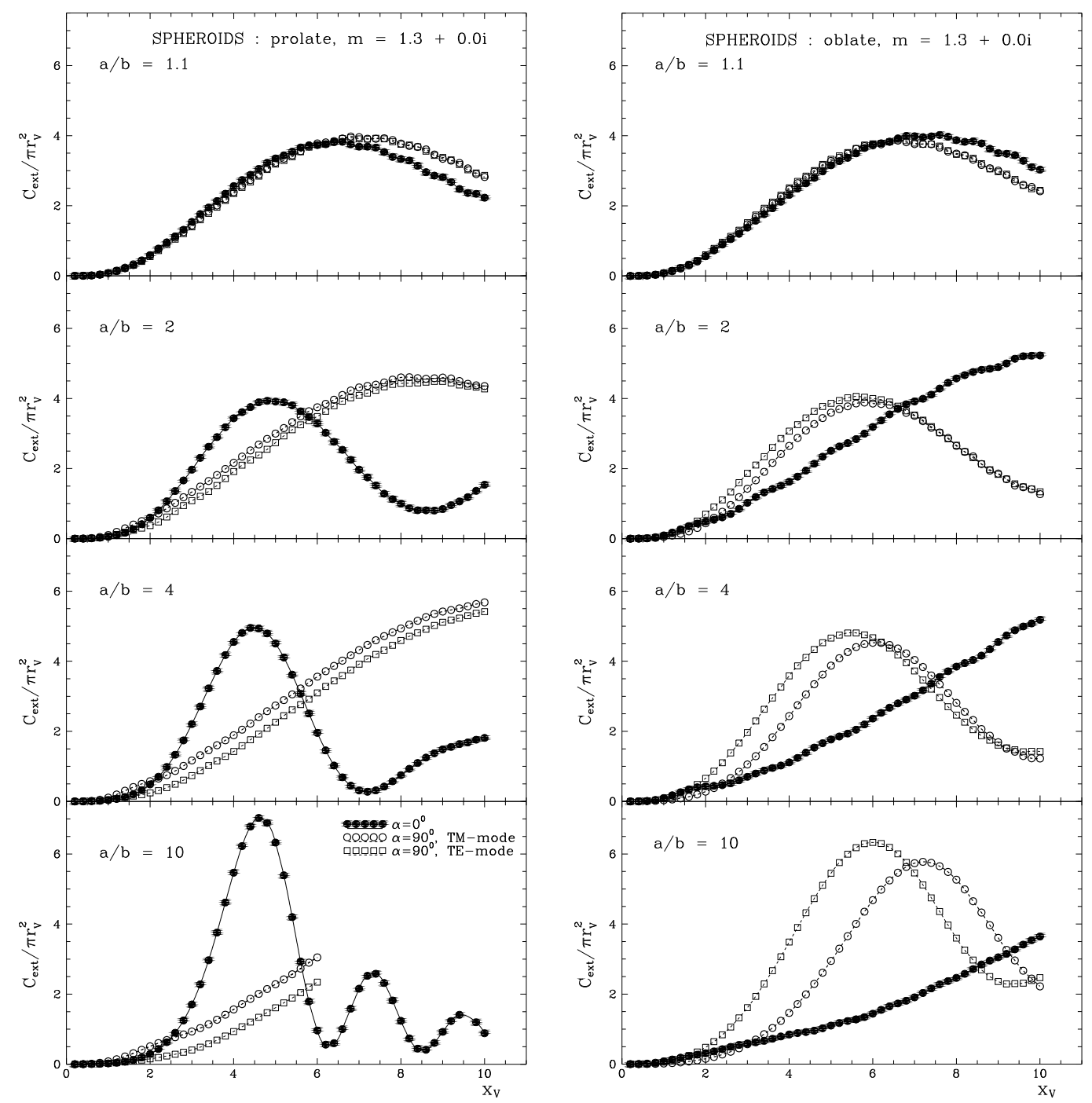

Fig. 2. Normalized extinction cross-sections vs $x_{V}$ for prolate and oblate spheroids with $m=1.3+0.0 i$.

presented as an example in Fig. 3, the behaviour of extinction is mainly determined by that of absorption.

The integral scattering properties of particles are characterized by their albedo (see Eq. (8)). This quantity depends on the particle size and, in general, on the particle shape. As far as we know the shape effects have not yet been discussed in the literature. Figure 5 shows the size dependence of the albedo for spheroids with $m=1.7+0.7 i$. The calculations were made for the particles with four aspect ratios $a / b=1.1,2,4,10$ and for two orientations $\alpha=0^{\circ}$ and $90^{\circ}$ (we adopt that the incident radiation is non-polarized).

It can be clearly seen that the albedo for non-spherical particles whose sizes are larger than a critical value $\left(x_{V} \approx 2-3\right)$ does not deviate more than $\sim 20 \%$ from that of spheres. The largest deviations in Fig. 5 occur for the aspect ratio 

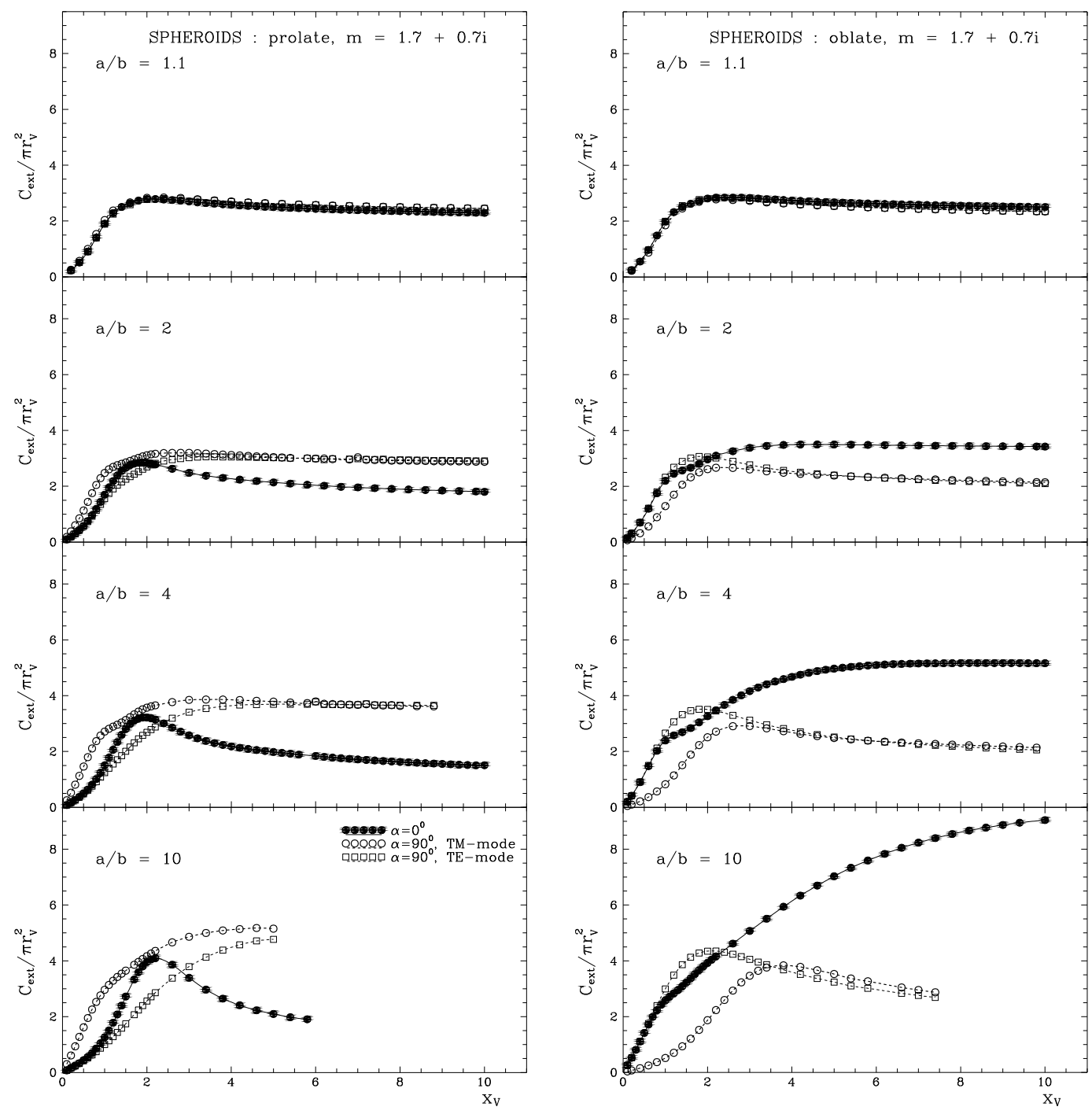

Fig. 3. Normalized extinction cross-sections vs $x_{V}$ for prolate and oblate spheroids with $m=1.7+0.7 i$.

$a / b=10$ and $\alpha=0^{\circ}$ (prolate spheroids) and $\alpha=90^{\circ}$ (oblate spheroids). Note that for arbitrarily oriented particles (3D-alignment), the distinction between the albedo of spheres and spheroids should be smaller (see Fig. 11 in (6) where the albedo for particles with $m=1.53+0.008 i$ and $a / b=2$ is plotted).

Our calculations made for particles with different absorption show that the distinction of the albedo for spheres and spheroidal particles remains rather small (within $\sim 20 \%$ ) if the ratio of the imaginary part of the refractive index to its real part $k / n \gtrsim 0.2-0.3$, which corresponds to $k \approx 0.3-0.4$ if $n=1.7$. Thus, we can conclude that the albedo of spheroidal particles from soot, graphite, iron very slightly depends on the particle shape. 

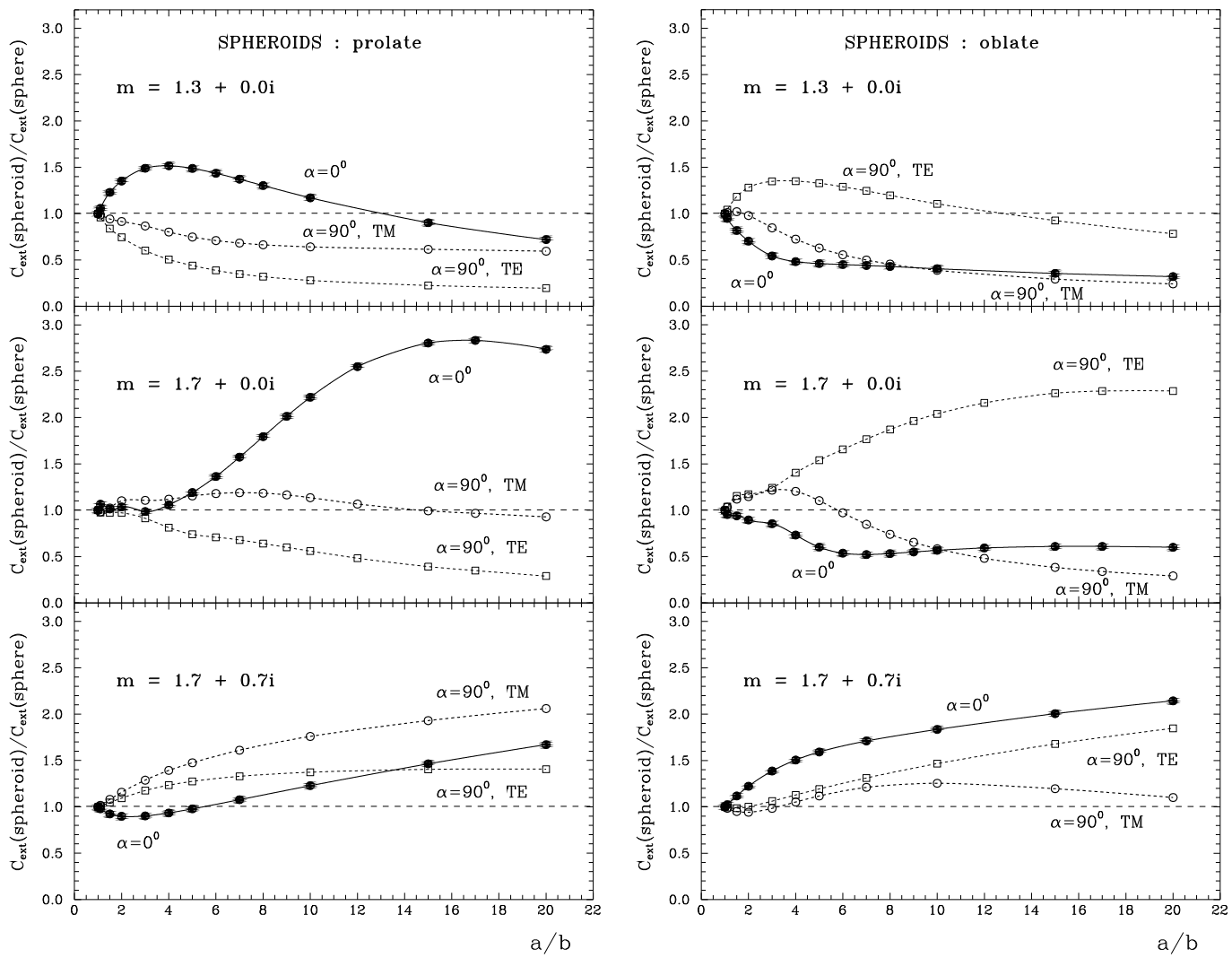

Fig. 4. Normalized extinction cross-sections (relative to that for equal volume sphere) vs $a / b$ for prolate and oblate spheroids with $x_{V}=3$ and $m=1.3+0.0 i, 1.7+0.0 i$, and $1.7+0.7 i$.

\subsection{Polarization}

If a volume contains aligned non-spherical particles, the initially non-polarized incident radiation will be partially polarized after passing the volume. The polarization degree can be used to estimate the alignment degree of the particles. The simplest and at the same time extreme case of particles' alignment is the perfect alignment of non-rotating particles (picked fence orientation). The maximum polarization usually occurs when the major axis of the particles is perpendicular to the direction of the incident radiation $\left(\alpha=90^{\circ}\right)$.

The previous calculations of the polarization (see (2; 9; 10) ) did not deal with large, highly absorbing particles. However, such particles exist in nature and can be aligned under terrestrial and extraterrestrial conditions.

The behaviour of the polarization efficiency $P / \tau$ (see Eq. (9)) for non-absorbing and absorbing spheroids is shown in Fig. 6 for the case when a maximum polarization is expected (picked fence alignment, $\alpha=90^{\circ}$ ). It is clearly seen from this Figure that the relatively large particles produce no polarization- 


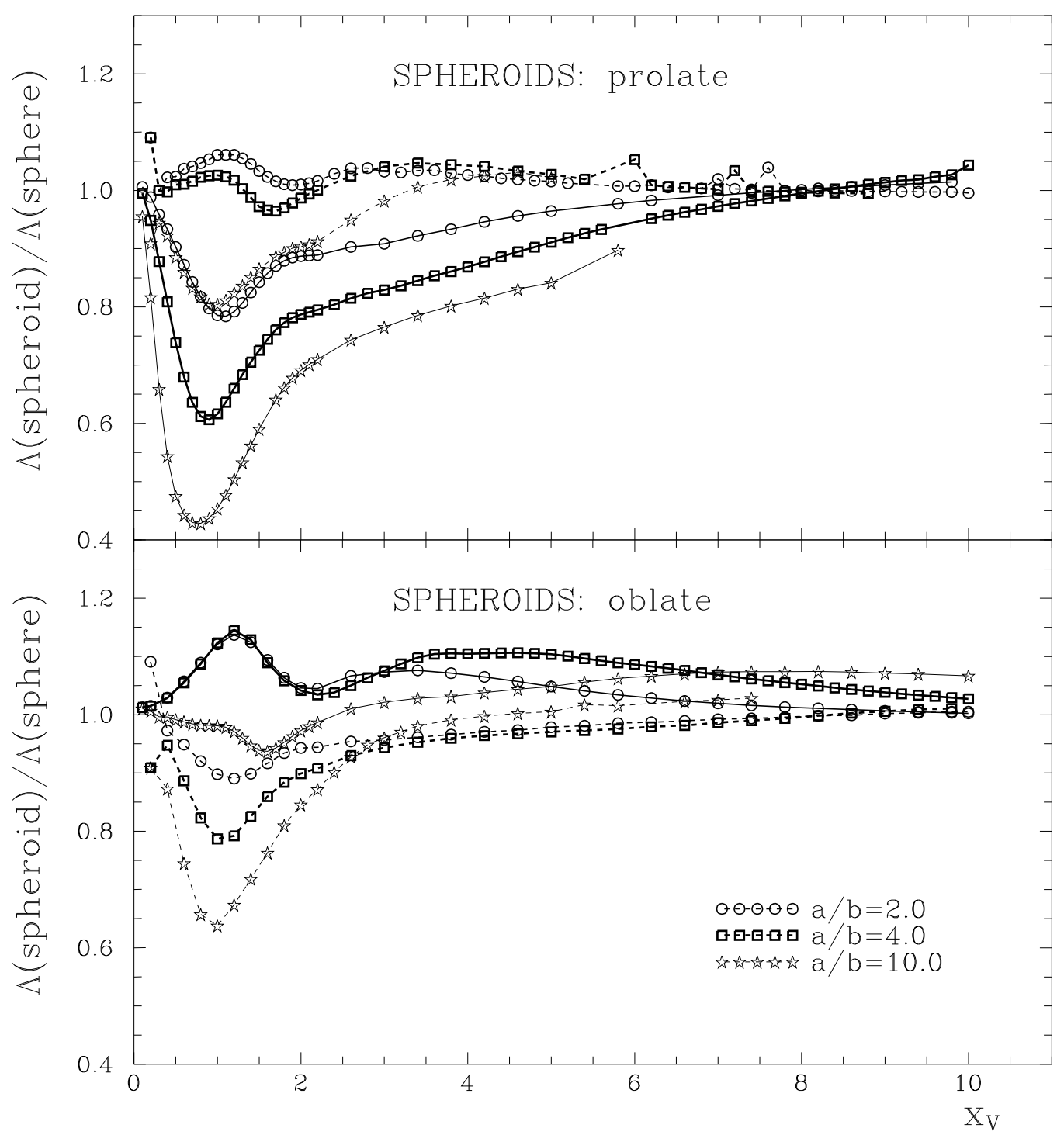

Fig. 5. Albedo of spheroidal particles with $m=1.7+0.7 i$ normalized relative to albedo of spherical particles with the same refractive index. The solid lines connect the points for $\alpha=0^{\circ}$, the dashed lines connect the points for $\alpha=90^{\circ}$.

independent of their shape. For absorbing particles, it occurs at smaller $x_{V}$ values than for non-absorbing particles. Note also that the polarization reversal takes place for disk-like particles. This effect seems to depend on the imaginary part of the refractive index as well.

\subsection{Benchmarks}

The results of computations for spheroids were previously published in the tabular form only in the papers of Voshchinnikov \& Farafonov (11; 14; 15) 

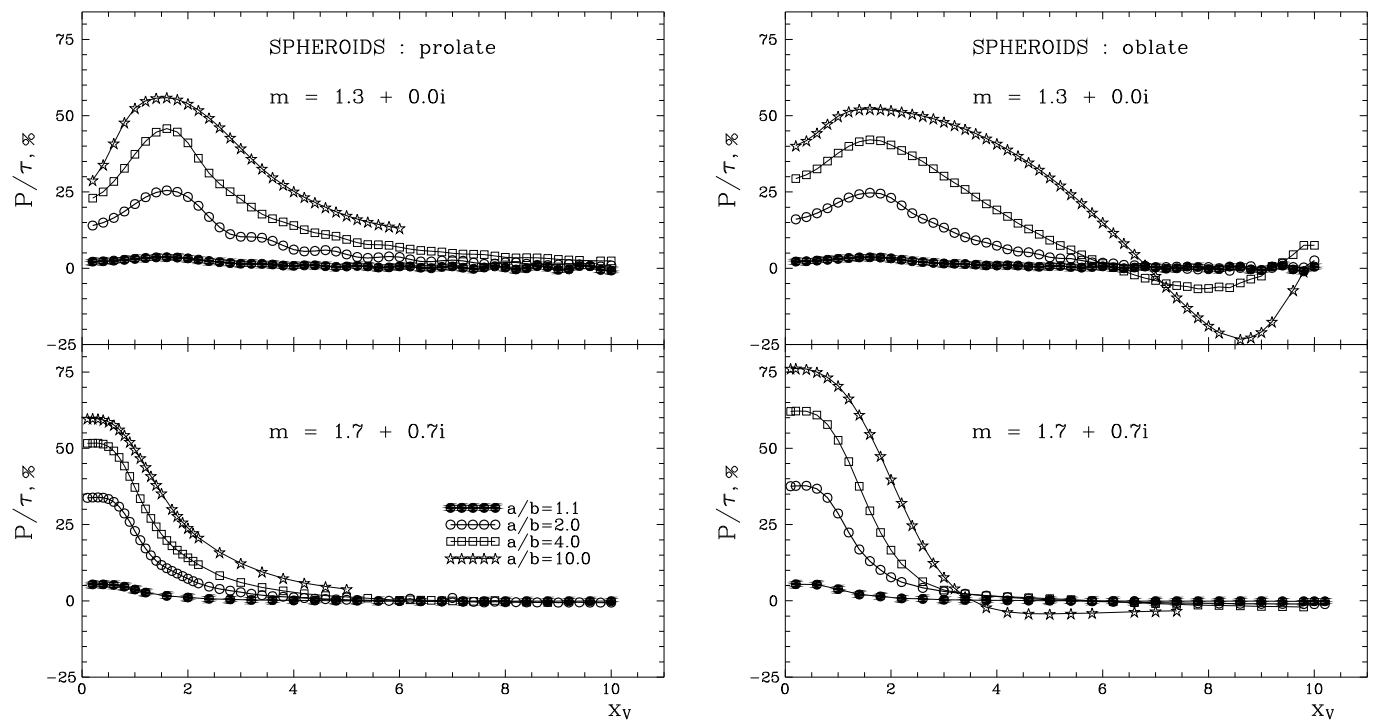

Fig. 6. Polarization efficiency vs $x_{V}$ for prolate and oblate spheroids with $m=1.3+0.0 i$ and $1.7+0.7 i, \alpha=90^{\circ}$.

(efficiency factors for spheroids with the refractive index $m=1.2$ ), Kuik et al. (16) and Hovenier et al. (4) (the scattering matrices for spheroids with $m=1.5+k i, k<0.01)$. Extensive calculations of the elements of the scattering matrices for randomly oriented slightly absorbing spheroids with $a / b \leq 2$ were performed by Mishchenko and others (see (6) and references therein).

In Tables 1 - 4, we present the normalized extinction and scattering crosssections for prolate and oblate spheroids with the refractive indices $m=2.5+$ $0.0 i, 1.7+0.7 i, 2.5+1.5 i$ and $3.0+4.0 i$. The results - all 7 (or in a few cases 6) significant digits - were obtained with at least two of four codes used (see Sects. 2.2-2.4).

\section{Editor: Tables $1-4$ are put here!!!}

The numbers printed by italics in Table 1 were calculated using the SVM2 code only. As the extinction and scattering cross-sections were the same (with 8 and more digits), the values presented in the Table are expected to be correct.

\section{Conclusions}

On the basis of the solutions to the light scattering problem by the separation of variables method and the T-matrix method we have considered the optical properties of absorbing spheroidal particles of different aspect ratios. 
Distinct approaches allowed to get the reliable results which may be used as benchmarks.

The following conclusions can be made from the study:

1. The extinction cross-sections for highly absorbing spheroids are typically 1.5 - 2 times larger than those for spheres of the same volume.

2. The albedo of non-spherical particles exhibits only a weak dependence on the particle shape and is determined mainly by the imaginary part of the refractive index.

3. For particles larger than a certain minimum size, the spheroidal particles do not polarize the transmitted radiation independent of their shape.

\section{Acknowledgements}

This work was supported by a grant from the Volkswagen Foundation.

\section{References}

[1] Asano, S. and Yamamoto, G., Light scattering by a spheroidal particle. Applied Optics 14 (1975) 29-49.

[2] Voshchinnikov, N.V. and Farafonov, V.G., Optical properties of spheroidal particles. Astrophysics and Space Science 204 (1993) 19-86.

[3] Barber, P.W. and Hill, S.C., Light Scattering by Particles: Computational Methods, World Scientific, Singapore, 1990.

[4] Michel B., Henning Th., Stognienko, R. and Rouleau, F., Extinction properties of dust grains: a new computational technique. Astrophysical J. 468 (1996) 834-841.

[5] Voshchinnikov, N.V., Electromagnetic scattering by homogeneous and coated spheroids: calculations using the separation of variables method. J. Quant. Spectrosc. Radiat. Transfer 55 (1996) 627-636.

[6] Mishchenko, M.I., Travis, L.D. and Mackowski, D.W., T-matrix computations of light scattering by nonspherical particles: a review. J. Quant. Spectrosc. Radiat. Transfer 55 (1996) 535-575.

[7] Hovenier, J.W., Lumme, K., Mishchenko, M.I., Voshchinnikov, N.V., Mackowski, D.W. and Rahola, J. Computations of scattering matrices of four types of non-spherical particles using diverse methods. J. Quant. Spectrosc. Radiat. Transfer 55 (1996) 695-705.

[8] Voshchinnikov, N.V., Il'in V.B. and Stognienko R., Comparative analysis of different solutions of light scattering problem for non-spherical particles. in Passive Infrared Remote Sensing of Clouds and the Atmosphere II, D.K. Lynch, Ed. Proc. SPIE. 2309, 1994, pp. 89-97. 
[9] Rogers, C. and Martin, P.G., On the shape of interstellar grains. Astrophysical J. 228 (1979) 450-464.

[10] Kim, S.-H. and Martin, P.G., The size distribution of interstellar dust particles as determined from polarization: spheroids. Astrophysical J. 444 (1995) 293-305.

[11] Voshchinnikov, N.V. and Farafonov, V.G., Light scattering by dielectric spheroids. I. Optics and Spectroscopy 58 (1985) 81-85.

[12] Farafonov, V.G., New method of calculations of light scattering by dielectric axisymmetric particles. Optics and Spectroscopy in press.

[13] Farafonov, V.G., Il'in, V.B. and Henning, Th., A new solution of the light scattering problem for axisymmetric particles. J. Quant. Spectrosc. Radiat. Transfer (1999) in press.

[14] Voshchinnikov, N.V. and Farafonov, V.G., Light scattering by dielectric spheroids. III. Optics and Spectroscopy 59 (1985) 750-753.

[15] Voshchinnikov, N.V. and Farafonov, V.G., Light scattering by dielectric spheroids. IV. Optics and Spectroscopy 60 (1986) 635-638.

[16] Kuik, F., de Haan, J.F. and Hovenier J.W., Benchmark results for single scattering by spheroids. J. Quant. Spectrosc. Radiat. Transfer 47 (1992) 477-489. 
Table 1: Normalized scattering cross-sections $C_{\text {sca }} / \pi r_{V}^{2}$ for spheroids with $m=2.5+0.0 i$

\begin{tabular}{|c|c|c|c|c|c|c|c|}
\hline \multirow[b]{2}{*}{$a / b$} & \multirow[b]{2}{*}{$x_{V}$} & \multicolumn{3}{|c|}{ Prolate spheroid } & \multicolumn{3}{|c|}{ Oblate spheroid } \\
\hline & & $\alpha=0^{\circ}$ & $\alpha=90^{\circ}(\mathrm{TM})$ & $\alpha=90^{\circ}(\mathrm{TE})$ & $\alpha=0^{\circ}$ & $\alpha=90^{\circ}(\mathrm{TM})$ & $\alpha=90^{\circ}(\mathrm{TE})$ \\
\hline \multirow[t]{4}{*}{2} & 0.1 & $8.132300 \mathrm{E}-05$ & $2.262901 \mathrm{E}-04$ & $8.162806 \mathrm{E}$ & 1.641502E-04 & $5.756336 \mathrm{E}-05$ & 1.637 \\
\hline & 0.3 & $6.610480 \mathrm{E}-03$ & $2.018734 \mathrm{E}-02$ & $6.819180 \mathrm{E}-03$ & $1.433111 \mathrm{E}-02$ & $4.685330 \mathrm{E}-03$ & -02 \\
\hline & 1.0 & $1.299951 \mathrm{E}-00$ & $3.736834 \mathrm{E}-00$ & $9.854978 \mathrm{E}-01$ & $2.464760 \mathrm{E}-00$ & $6.647633 \mathrm{E}-01$ & $3 \mathrm{E}-00$ \\
\hline & 3.0 & $2.756951 \mathrm{E}-00$ & $2.423317 \mathrm{E}-00$ & $1.456577 \mathrm{E}-00$ & $4.484763 \mathrm{E}-00$ & $1.777398 \mathrm{E}-00$ & $3.846601 \mathrm{E}-00$ \\
\hline \multirow[t]{4}{*}{4} & 0.1 & $6.871662 \mathrm{E}-05$ & 4.2 & & $2.612223 \mathrm{I}$ & & 2.6000 \\
\hline & 0.3 & $5.105309 E-03$ & $E-02$ & 5.6 & 2.307 & $2.874841 E-03$ & 2.217663E-02 \\
\hline & 1.0 & $7.055768 E-01$ & $5.493155 E-00$ & $5.426740 E-01$ & $3.362340 E-00$ & 2.595873E-01 & $5.360019 E-00$ \\
\hline & 3.0 & $1.673046 E-00$ & 5.755844E-00 & 4.730299E-00 & $7.392485 E-00$ & 2.580128E-00 & 3.791884E-00 \\
\hline \multirow[t]{4}{*}{10} & 0.1 & 6.058991E-05 & $6.745045 E-04$ & $E-05$ & $4.423506 E-04$ & 2.643817E-05 & $4.383382 E-04$ \\
\hline & 0.3 & $3.446015 E-03$ & 5.950350E-02 & 4.588377E-03 & 3.852378E-02 & 1.918230E-03 & $3.569470 E-02$ \\
\hline & 1.0 & $3.088317 E-01$ & $6.153316 E-00$ & 2.283901E-01 & $3.605985 E-00$ & $1.053865 E-01$ & 5.126838E-00 \\
\hline & 3.0 & $4.612520 E-01$ & 9.781859E-00 & $7.081355 E-00$ & 7.766600E-00 & $7.497780 E-00$ & $4.701476 E-00$ \\
\hline
\end{tabular}


Table 2: Normalized extinction and scattering cross-sections $C_{\text {ext, sca }} / \pi r_{V}^{2}$ for spheroids with $m=1.7+0.7 i$

\begin{tabular}{|c|c|c|c|c|c|c|c|}
\hline \multirow[b]{2}{*}{$a / b$} & \multirow[b]{2}{*}{$x_{V}$} & \multicolumn{2}{|c|}{$\alpha=0^{\circ}$} & \multicolumn{4}{|c|}{$\alpha=90^{\circ}$} \\
\hline & & $C_{\mathrm{ext}} / \pi r_{V}^{2}$ & $C_{\mathrm{sca}} / \pi r_{V}^{2}$ & $C_{\text {ext }}^{\mathrm{TM}} / \pi r_{V}^{2}$ & $C_{\mathrm{sca}}^{\mathrm{TM}} / \pi r_{V}^{2}$ & $C_{\mathrm{ext}}^{\mathrm{TE}} / \pi r_{V}^{2}$ & $C_{\mathrm{sca}}^{\mathrm{TE}} / \pi r_{V}^{2}$ \\
\hline \multicolumn{8}{|c|}{ Prolate spheroid } \\
\hline \multirow[t]{5}{*}{2} & 0.1 & $9.260996 \mathrm{E}-02$ & $6.520100 \mathrm{E}-05$ & $1.867292 \mathrm{E}-01$ & $1.323250 \mathrm{E}-04$ & $9.250492 \mathrm{E}-02$ & $6.544660 \mathrm{E}-05$ \\
\hline & 0.3 & E-01 & & & $6 \mathrm{E}-02$ & & -03 \\
\hline & 1.0 & $2 \mathrm{E}-00$ & 3.8 & 2.47 & $288 \mathrm{E}-01$ & 1.556 & -01 \\
\hline & 3.0 & $\mathrm{E}-00$ & & & $5 \mathrm{E}-00$ & & -00 \\
\hline & 10.0 & $1.804330 \mathrm{E}-00$ & $9.737252 \mathrm{E}-01$ & $2.874307 \mathrm{E}-00$ & $1.607439 \mathrm{E}-00$ & $2.908106 \mathrm{E}-00$ & $1.453882 \mathrm{E}-00$ \\
\hline \multirow[t]{4}{*}{4} & 0.1 & $8.155853 \mathrm{E}-02$ & $5.681280 \mathrm{E}-05$ & $2.550670 \mathrm{E}-01$ & $1.807060 \mathrm{E}-04$ & 8.14151 & $5.748690 \mathrm{E}-05$ \\
\hline & 0.3 & 2.62 & 4.1 & 8.11 & $5 \mathrm{E}-02$ & & 4.56 \\
\hline & 1.0 & E-00 & 2.66 & 9E-00 & $7 \mathrm{E}-01$ & 1.243 & 3.19 \\
\hline & 3.0 & $2.580295 \mathrm{E}-00$ & $9.865859 \mathrm{E}-01$ & 3.848184E-00 & $1.966160 \mathrm{E}-00$ & 3.40958 E-00 & $1.515164 \mathrm{E}-00$ \\
\hline \multirow[t]{3}{*}{10} & 0.1 & 7.602 & & & $2.122690 \mathrm{E}$ & & -05 \\
\hline & 0.3 & 2.39 & & $9.378379 \mathrm{E}-01$ & $1.631801 \mathrm{E}-02$ & & $\mathrm{E}-03$ \\
\hline & & & & Oblat & & & \\
\hline \multirow[t]{4}{*}{2} & 0.1 & $1.533476 \mathrm{E}-01$ & $1.086960 \mathrm{E}-04$ & $6.964599 \mathrm{E}-02$ & 4.913110E-05 & $1.534705 \mathrm{E}-01$ & $1.084390 \mathrm{E}-04$ \\
\hline & 0.3 & $4.941085 \mathrm{E}-01$ & 9.02 & 01 & 3.938983E-03 & 4.971 & $8.843852 \mathrm{E}-03$ \\
\hline & 1.0 & E-00 & 7.1 & 00 & 3.1 & 2.3 & $\mathrm{E}-01$ \\
\hline & 3.0 & $3.378955 \mathrm{E}-00$ & $1.672422 \mathrm{E}-00$ & $2.603296 \mathrm{E}-00$ & $1.040019 \mathrm{E}-00$ & $2.768031 \mathrm{E}-00$ & $1.330862 \mathrm{E}-00$ \\
\hline \multirow[t]{3}{*}{4} & 0.1 & $2.021662 \mathrm{E}-01$ & $1.432990 \mathrm{E}-04$ & 4.737106E-02 & $3.331120 \mathrm{E}-05$ & $2.023435 \mathrm{E}-01$ & 1.426270E-04 \\
\hline & 0.3 & $6.442415 \mathrm{E}-01$ & & & $2.574738 \mathrm{E}-03$ & & $7 \mathrm{E}-02$ \\
\hline & 1.0 & $2.394211 \mathrm{E}-00$ & $7.756880 \mathrm{E}-01$ & $8.254487 \mathrm{E}-01$ & $1.707659 \mathrm{E}-01$ & $2.658211 \mathrm{E}-00$ & $6.204606 \mathrm{E}-01$ \\
\hline \multirow[t]{2}{*}{10} & 0.1 & 2.59201 E-01 & $1.833420 \mathrm{E}-04$ & 3.51934 E-02 & $2.458170 \mathrm{E}-05$ & 2.59355 E-01 & 1.816740E-04 \\
\hline & 0.3 & 8.101747E-01 & $1.452893 \mathrm{E}-02$ & $1.102168 \mathrm{E}-01$ & $1.774836 \mathrm{E}-03$ & 8.13450 E-01 & 1.343542E-02 \\
\hline
\end{tabular}


Table 3: Normalized extinction and scattering cross-sections $C_{\text {ext, sca }} / \pi r_{V}^{2}$ for spheroids with $m=2.5+1.5 i$

\begin{tabular}{|c|c|c|c|c|c|c|c|}
\hline \multirow[b]{2}{*}{$a / b$} & \multirow[b]{2}{*}{$x_{V}$} & \multicolumn{2}{|c|}{$\alpha=0^{\circ}$} & \multicolumn{4}{|c|}{$\alpha=90^{\circ}$} \\
\hline & & $C_{\text {ext }} / \pi r_{V}^{2}$ & $C_{\text {sca }} / \pi r_{V}^{2}$ & $C_{\mathrm{ext}}^{\mathrm{TM}} / \pi r_{V}^{2}$ & $C_{\mathrm{sca}}^{\mathrm{TM}} / \pi r_{V}^{2}$ & $C_{\mathrm{ext}}^{\mathrm{TE}} / \pi r_{V}^{2}$ & $C_{\mathrm{sca}}^{\mathrm{TE}} / \pi r_{V}^{2}$ \\
\hline \multicolumn{8}{|c|}{ Prolate spheroid } \\
\hline \multirow[t]{4}{*}{2} & 0.1 & 7.015863E-02 & $1.324916 \mathrm{E}-04$ & $2.555773 \mathrm{E}-01$ & $4.901860 \mathrm{E}-04$ & $6.978007 \mathrm{E}-02$ & $1.329890 \mathrm{E}-04$ \\
\hline & 0.3 & $2.606615 \mathrm{E}-01$ & $1.085718 \mathrm{E}-02$ & $9.351659 \mathrm{E}-01$ & 4.418671E-02 & $2.499507 \mathrm{E}-01$ & $1.120452 \mathrm{E}-02$ \\
\hline & 1.0 & $2.545641 \mathrm{E}-00$ & $8.269798 \mathrm{E}-01$ & $4.506818 \mathrm{E}-00$ & $2.290117 \mathrm{E}-00$ & $2.378954 \mathrm{E}-00$ & $1.018659 \mathrm{E}-00$ \\
\hline & 3.0 & $2.543020 \mathrm{E}-00$ & $1.196810 \mathrm{E}-00$ & $3.377612 \mathrm{E}-00$ & $2.030276 \mathrm{E}-00$ & $3.018778 \mathrm{E}-00$ & $1.561478 \mathrm{E}-00$ \\
\hline \multirow[t]{4}{*}{4} & 0.1 & 5.77412 E-02 & $1.079560 \mathrm{E}-04$ & 5.61456 E-01 & $1.078514 \mathrm{E}-03$ & $5.73428 \mathrm{E}-02$ & $1.092344 \mathrm{E}-04$ \\
\hline & 0.3 & 2.09854 E-01 & 8.054995E-03 & $1.994433 \mathrm{E}-00$ & $9.529425 \mathrm{E}-02$ & 1.99005 E-01 & 8.869991E-03 \\
\hline & 1.0 & 2.23898 E-00 & $5.318469 \mathrm{E}-01$ & $4.844781 \mathrm{E}-00$ & $2.443652 \mathrm{E}-00$ & $1.709826 \mathrm{E}-00$ & $6.654597 \mathrm{E}-01$ \\
\hline & 3.0 & $2.564187 \mathrm{E}-00$ & $9.810827 \mathrm{E}-01$ & 4.17736 E-00 & $2.576599 \mathrm{E}-00$ & $3.444722 \mathrm{E}-00$ & $1.733285 \mathrm{E}-00$ \\
\hline \multirow[t]{2}{*}{10} & 0.1 & 5.18757 E-02 & $9.344730 \mathrm{E}-05$ & 8.83893 E-01 & $1.689619 \mathrm{E}-03$ & 5.15928 E-02 & $9.740630 \mathrm{E}-05$ \\
\hline & \multicolumn{7}{|c|}{ Oblate spheroid } \\
\hline \multirow[t]{4}{*}{2} & 0.1 & $1.683208 \mathrm{E}-01$ & $3.228280 \mathrm{E}-04$ & $4.590543 \mathrm{E}-02$ & 8.685830E-05 & $1.690525 \mathrm{E}-01$ & $3.220660 \mathrm{E}-04$ \\
\hline & 0.3 & $6.046926 \mathrm{E}-01$ & $2.854920 \mathrm{E}-02$ & $1.682741 \mathrm{E}-01$ & $7.112129 \mathrm{E}-03$ & 6.24682 E-01 & $2.798368 \mathrm{E}-02$ \\
\hline & 1.0 & $4.007697 \mathrm{E}-00$ & $2.005398 \mathrm{E}-00$ & $1.852325 \mathrm{E}-00$ & $6.182319 \mathrm{E}-01$ & $3.928646 \mathrm{E}-00$ & $1.726163 \mathrm{E}-00$ \\
\hline & 3.0 & $3.644115 \mathrm{E}-00$ & $2.139153 \mathrm{E}-00$ & $2.436512 \mathrm{E}-00$ & $1.057701 \mathrm{E}-00$ & $2.916411 \mathrm{E}-00$ & $1.654727 \mathrm{E}-00$ \\
\hline \multirow[t]{3}{*}{4} & 0.1 & 3.07216 E-01 & $5.903690 \mathrm{E}-04$ & $2.714797 \mathrm{E}-02$ & $5.130230 \mathrm{E}-05$ & $3.085216 \mathrm{E}-01$ & $5.876060 \mathrm{E}-04$ \\
\hline & 0.3 & $1.089947 \mathrm{E}-00$ & $5.221910 \mathrm{E}-02$ & $9.612426 \mathrm{E}-02$ & $4.009507 \mathrm{E}-03$ & $1.123093 \mathrm{E}-00$ & $5.017062 \mathrm{E}-02$ \\
\hline & 1.0 & $4.550598 \mathrm{E}-00$ & $2.313767 \mathrm{E}-00$ & 1.04303 E-00 & $2.876447 \mathrm{E}-01$ & 4.68404 E-00 & $1.847488 \mathrm{E}-00$ \\
\hline
\end{tabular}


Table 4: Normalized extinction and scattering cross-sections $C_{\text {ext, sca }} / \pi r_{V}^{2}$ for spheroids with $m=3.0+4.0 i$

\begin{tabular}{|c|c|c|c|c|c|c|c|}
\hline \multirow[b]{2}{*}{$a / b$} & \multirow[b]{2}{*}{$x_{V}$} & \multicolumn{2}{|c|}{$\alpha=0^{\circ}$} & \multicolumn{4}{|c|}{$\alpha=90^{\circ}$} \\
\hline & & $C_{\text {ext }} / \pi r_{V}^{2}$ & $C_{\text {sca }} / \pi r_{V}^{2}$ & $C_{\text {ext }}^{\mathrm{TM}} / \pi r_{V}^{2}$ & $C_{\mathrm{sca}}^{\mathrm{TM}} / \pi r_{V}^{2}$ & $C_{\mathrm{ext}}^{\mathrm{TE}} / \pi r_{V}^{2}$ & $C_{\mathrm{sca}}^{\mathrm{TE}} / \pi r_{V}^{2}$ \\
\hline \multicolumn{8}{|c|}{ Prolate spheroid } \\
\hline \multirow[t]{5}{*}{2} & 0.1 & $3.459049 \mathrm{E}-02$ & $1.836889 \mathrm{E}-04$ & 1.929191E-01 & $1.115412 \mathrm{E}-03$ & $3.339258 \mathrm{E}-02$ & $1.843746 \mathrm{E}-04$ \\
\hline & 0.3 & $1.944302 \mathrm{E}-01$ & $1.537309 \mathrm{E}-02$ & $9.098178 \mathrm{E}-01$ & $1.129382 \mathrm{E}-02$ & $1.661101 \mathrm{E}-01$ & $1.585916 \mathrm{E}-02$ \\
\hline & 1.0 & $1.894857 \mathrm{E}-00$ & $9.206887 \mathrm{E}-01$ & $5.495079 \mathrm{E}-00$ & $3.970900 \mathrm{E}-00$ & $2.283547 \mathrm{E}-00$ & $1.438695 \mathrm{E}-00$ \\
\hline & 3.0 & $2.388466 \mathrm{E}-00$ & $1.478325 \mathrm{E}-00$ & $3.453263 \mathrm{E}-00$ & $2.702600 \mathrm{E}-00$ & $2.762240 \mathrm{E}-00$ & $1.941164 \mathrm{E}-00$ \\
\hline & \multicolumn{7}{|c|}{ Oblate spheroid } \\
\hline 2 & 0.1 & $1.017897 \mathrm{E}-01$ & $5.864570 \mathrm{E}-04$ & $2.106561 \mathrm{E}-02$ & $1.116025 \mathrm{E}-04$ & $1.046258 \mathrm{E}-01$ & $5.850970 \mathrm{E}-04$ \\
\hline & 0.3 & $4.583023 \mathrm{E}-01$ & $5.527781 \mathrm{E}-02$ & $1.197910 \mathrm{E}-01$ & $9.258967 \mathrm{E}-03$ & $5.167953 \mathrm{E}-01$ & $5.426552 \mathrm{E}-02$ \\
\hline & 1.0 & $5.236749 \mathrm{E}-00$ & $3.731015 \mathrm{E}-00$ & $1.404715 \mathrm{E}-00$ & $6.821323 \mathrm{E}-01$ & $4.215200 \mathrm{E}-00$ & $2.800509 \mathrm{E}-00$ \\
\hline & 3.0 & $3.647681 \mathrm{E}-00$ & $2.814034 \mathrm{E}-00$ & $2.009705 \mathrm{E}-00$ & $1.173967 \mathrm{E}-00$ & $3.040199 \mathrm{E}-00$ & $2.239816 \mathrm{E}-00$ \\
\hline
\end{tabular}

